\title{
Computational Study of Collective Cell Migration By Meshfree Method
}

\author{
Jie Bai ${ }^{1, \#, ~ L i q i a n g ~ L i n ~}{ }^{1, \#}$ and Xiaowei Zeng, ${ }^{1, *}$
}

\begin{abstract}
The collective cell migration behavior on a substrate was studied using RKPM meshfree method. The cells were modeled as nematic liquid crystal with hyperelastic cell nucleus. The cell-substrate and cell-cell interactions were modeled by coarse-grained potential forces. Through this study, the pulling and pushing phenomenon during collective cell migration process was observed and it was found that the individual cell mobility significantly influenced the collective cell migratory behavior. More self-propelled cells are in the system along the same direction, the faster the collective group migrates toward coordinated direction. The parametric study on cell-cell adhesion strength indicated that as the adhesion strength increases, the collective cell migration speed increases. It also showed that the mechanical stress in leader cell is higher than stress in follower cells.
\end{abstract}

Keywords: Cell-cell interaction, cell-substrate interaction, collective cell migration, meshfree method, numerical simulation.

\section{Introduction}

Cell migration plays a significant role in many biological processes at different stages throughout the entire life of creatures [Frascoli, Hughes, Zaman et al. (2013); Li and Sun (2014); Hwang, Jung, Lee et al. (2015)]. Many biological activities require the coordinated cell movement toward a certain direction in a collective manner. Collective cell migration happens during tumor matastasis, wound healing process, morphogenesis of different organs or tissues, immune response, etc. For instance, during the inflammatory response, the leukocytes will move through the endothelial borders toward the site of infection in a highly coordinated manner in animal immune system [Simon and Green (2005); Muller (2013); Orellana, García-Solares, Donnez et al. (2017)].

Migratory behavior of cell group could be influenced by the interaction between neighboring cells and the interaction between cell and the extracellular matrix. In order to study cell motions in a collective manner and to investigate their roles in fundamental biological process, a number of studies have been conducted. The specific genes and key modules that influence cell migration and cell adhesion now can be identified by the highthroughput genomic methods [Simpson, Selfors, Bui et al. (2008); Vitorino and Meyer (2008); Wu, Siadaty, Berens et al. (2008)]. These studies showed that different genes or proteins regulate collective cell behavior in different ways. Several studies found that the

\footnotetext{
${ }^{1}$ Department of Mechanical Engineering, University of Texas at San Antonio, San Antonio, TX 78249, USA.

\# These two authors contributed equally to this work.

${ }^{*}$ Corresponding Author: Xiaowei Zeng. Email: xiaowei.zeng@utsa.edu.
} 
adhesion of cell-cell junction which results from the intercellular transmission of mechanical forces would turn the highly random and uncoordinated cell motion into a coordinated collective motion [Vitorino and Meyer (2008); Vedula, Leong, Lai et al. (2012)]. Tambe et al. [Tambe, Hardin, Angelini et al. (2011)] found that cell-cell interaction forces govern the collective migration, directing cells moving along the orientations of minimal intercellular shear stress.

Meanwhile, a lot of recent studies have been focused on theoretical modeling of the collective cell migratory processes. Some discrete mathematical models such as vertex models [Honda and Eguchi (1980)] and cellular potts models [Graner and Glazier (1992)] have been developed and extensively used in the numerical simulation for cell migration and many other biological processes. These models take into account some features such as cell elasticity, contractility and cell-cell adhesion, and identify that intercellular forces regulate the cell shape [Farhadifar, Röper, Aigouy et al. (2007); Fletcher, Osterfield, Baker et al. (2014)]. By employing a vertex model, Bi et al. [Bi, Lopez, Schwarz et al. (2015)] found a new type of liquid-to-solid transition in confluent tissues. Continuum models have also been used to study the collective cell migration processes, where the cell sheet is considered as active continuous media. Chen et al. [Chen and Brodland (2000)] and Brodland et al. [ Brodland, Viens and Veldhuis (2007)] proposed cell-based finite element models to study the stretching of cell sheet. A finite element method incorporating cell-cell interfacial interaction was used to study the collective epithelial migration [Lin and Zeng (2018)]. It was found that normal cell-cell adhesion regulates the coordinated epithelial movements.

Although the collective cell migratory behaviors and their roles in various physiological and pathological processes have been extensively studied, the mechanisms of coordination among cells are still not well understood due to the lack of advanced computational tools. How the mechanical clues will influence the collective cell migration behavior is not very clear. To elucidate how individual cell mobility and cell-cell interaction will affect the collective migratory behavior, we model the cell aggregates containing three monocytes to study the collective cell migration. In this study, we use liquid crystal and hyperelastic material models to model major cell components. A coarse-grained potential force was used to represent the cell-cell/substrate interactions. The RKPM meshfree method was employed to study the collective cell migratory behavior.

\section{Computational algorithms}

The numerical simulations were conducted by using the Reproducing Kernel Particle Methods (RKPM) [Liu, Li and Belytschko (1997); Li and Liu (2002)]. A total Lagrangian meshfree formulation was developed under finite deformation and a related Galerkin weak formulation was derived for numerical computation [Lin and Zeng (2017)]:

$$
\begin{aligned}
& \int_{\Omega_{0}} \rho_{0} \ddot{\boldsymbol{u}} \delta \boldsymbol{u} d \Omega=\int_{\Omega_{0}} \rho_{0} \boldsymbol{B} \delta \boldsymbol{u} d \Omega+\int_{\Gamma_{t}} \boldsymbol{f}^{\boldsymbol{c}-\boldsymbol{s}} \delta \boldsymbol{u} d \mathrm{~S}+\int_{\Gamma_{t}} \boldsymbol{f}^{\boldsymbol{c}-\boldsymbol{c}} \delta \boldsymbol{u} d \mathrm{~S}+\int_{\Gamma_{t}} \overline{\boldsymbol{T}} \delta \boldsymbol{u} d \mathrm{~S}- \\
& \int_{\Omega_{0}} \boldsymbol{P} \delta \boldsymbol{F} d \Omega
\end{aligned}
$$

where $\Omega_{0}, \rho_{0}$ represent the volume and material density in the reference configuration respectively; $\Gamma_{t}$ is external traction boundary; $\boldsymbol{P}$ is the first Piola-Kirchhoff stress tensor; $\boldsymbol{F}$ is the deformation gradient; $\boldsymbol{B}$ is the body force density; $\overline{\boldsymbol{T}}$ defines the external driving traction 
vector; $\boldsymbol{f}^{\boldsymbol{c}-\boldsymbol{s}}$ and $\boldsymbol{f}^{\boldsymbol{c}-\boldsymbol{c}}$ are the cell-substrate and cell-cell interaction forces. The discrete equations of motion can be derived following the standard meshfree discretization procedure:

$\boldsymbol{M} \ddot{\boldsymbol{u}}=\boldsymbol{F}^{b}+\boldsymbol{F}^{c-s}+\boldsymbol{F}^{c-c}+\boldsymbol{F}^{d}-\boldsymbol{F}^{i n t}$

where $\boldsymbol{u}$ is the displacement; $\boldsymbol{F}^{b}$ is the body force; $\boldsymbol{F}^{c-s}$ is the cell-substrate interaction force; $\boldsymbol{F}^{c-c}$ is the cell-cell interaction force; $\boldsymbol{F}^{d}$ is the external driving force (e.g., protrusion force applied at the cell leading edge); $\boldsymbol{F}^{\text {int }}$ denotes internal force array arising from the current state of stress. The detailed expression of the forces can be found in Lin et al. [Lin and Zeng (2017)].

\section{Model description}

In this study, three monocytes were placed on a ligand-coated substrate surface with the right cell defined as Cell-1, the middle cell defined as Cell-2, and the left cell defined as Cell-3. The cell protrusion force will drive cell moving on the substrate. A schematic diagram of collective cell migration on the substrate is shown in Fig. 1 below.

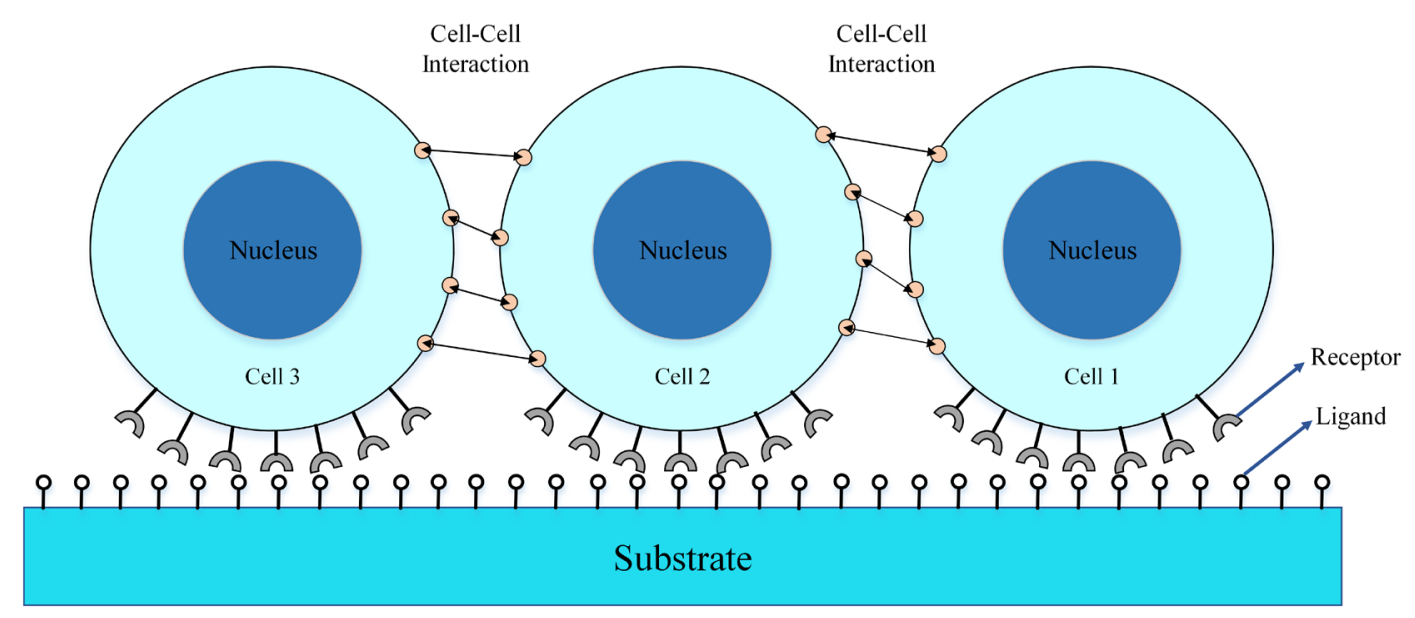

Figure 1: Schematic diagram of collective cell migration on a substrate

\subsection{Cell-substrate interaction}

At the beginning of the cell migration process, the cells will adhere to the substrate surface by forming the interaction bonds with the substrate. After that, the cells will spread over the substrate and finally move along the substrate collectively. Many theoretical models have been developed and used in order to capture the ligand-receptor interaction behavior [Zhu (2000); Zhu, Bao and Wang (2000); Liu, Zhang, Cheng et al. (2007); Sauer and Li (2007); Farsad and Vernerey (2012); Ruiz-Herrero, Estrada, Guantes et al. (2013); Fan and Li (2015)]. Here in this paper, a two-parameter potential given by Seifert [Seifert (1991)] was used to represent the cell-substrate interaction. Roy et al. [Roy and Qi (2010)] used this potential to study the cell crawling phenomenon. This adhesive potential shown in Eq. (3) was also used by Lin et al. [Lin and Zeng (2017)] to study the cell rolling behavior in a flow channel: 
$U(r)=\sigma\left[\left(\frac{\varepsilon}{r}\right)^{4}-2\left(\frac{\varepsilon}{r}\right)^{2}\right]$

where $\sigma$ is the energy depth; $r$ represents the distance between the cell surface and the ECM substrate. Thus, the adhesive force vector can be derived by taking the negative gradient of the potential function:

$\boldsymbol{F}(r)=-\frac{\partial U}{\partial \boldsymbol{r}}=\frac{4 \sigma}{\varepsilon}\left[\left(\frac{\varepsilon}{r}\right)^{5}-\left(\frac{\varepsilon}{r}\right)^{3}\right] \frac{r}{r}$

Taken into account the ligand-receptor bond density, the cell-substrate adhesive force can be derived as in Eq. (5) below. Here $N_{c-s}$ is the surface bond density between the cell and substrate.

$\boldsymbol{f}^{c-s}(r)=N_{c-s} \boldsymbol{F}(r)=N_{c-s} \frac{4 \sigma}{\varepsilon}\left[\left(\frac{\varepsilon}{r}\right)^{5}-\left(\frac{\varepsilon}{r}\right)^{3}\right] \frac{r}{r}$

\subsection{Cell-cell interaction model}

Unlike the single cell moving on the substrate, collective cell migration is a more complicated process which include how cells interact with the substrate, also how a cell will interact with its neighbors. Here in this work, the cell-cell interaction was also represented by an adhesive potential function, which is similar to the cell-substrate interaction described in Eq. (5). The cell-cell interaction force may be calculated as in Eq. (6) below. Here $N_{c-c}$ is the surface bond density between two neighboring cells.

$\boldsymbol{f}^{c-c}(r, x)=N_{c-c} \boldsymbol{F}(r)=N_{c-c} \frac{4 \sigma}{\varepsilon}\left[\left(\frac{\varepsilon}{r}\right)^{5}-\left(\frac{\varepsilon}{r}\right)^{3}\right] \frac{r}{r}$

\subsection{Soft matter cell model}

Different types of cells have different structures, and the mechanical properties of cells is a continuing topic of debate in the field of biophysics. Many experimental techniques such as atomic force microscopy (AFM) [Haase and Pelling (2015)], shearing and compression tests [Thoumine, Ott, Cardoso et al. (1999)], magnetic tweezers [Sleep, Wilson, Simmons et al. (1999); Dao, Lim and Suresh (2003)], optical dynamic light scattering and diffusing [Peetermans, Matthews, Nishio et al. (1987)] have been used to help probing the cell mechanics. Local viscoelasticity can be observed at different regions of cell with different stiffness. Recent studies found that cells can undergo a phase transition between fluid-like and solid-like states [Munder, Midtvedt, Franzmann et al. (2016); Mongera, Rowghanian, Gustafson et al. (2018)]. In the meantime, the theoretical modeling of cytoskeleton medium has been conducted using a variety of material models such as hyperelastic models [McGarry, Murphy and McHugh (2005); Mihai, Wyatt and Goriely (2017)], viscoelastic models [Karcher, Lammerding, Huang et al. (2003); Ragazzon, Gravdahl and Vagia (2018)], liquid crystal models [Helfrich (1973); Rey (2010); Zeng and Li (2011)], active gel models [Kruse, Joanny, Jülicher et al. (2005); Prost, Jülicher and Joanny (2015)], and multiphasic models [Vernerey and Farsad (2011); Zeng and Li (2012)].

Cell nucleus plays a pivotal role in sensing external conditions and force transfer. Experimental measurements have shown that the nucleus stiffness is nearly 10 times greater than the stiffness of cytoplasm [Thoumine and Ott (1997); Caille, Thoumine, Tardy 
et al. (2002)]. Thus, in this work, we use a soft matter cell model developed by Zeng and $\mathrm{Li}$ [Zeng and $\mathrm{Li}(2012)]$ to study the collective cell migratory behavior. The cytoplasm and cytoskeleton network was modeled as nematic liquid crystal material which was represented by a simplified version of Ericksen-Leslie theory [Lin and Liu (2000)]. The Cauchy stress is described in Zeng et al. [Zeng and Li (2012)] as below:

$\sigma_{i j}=-p \delta_{i j}+2 \mu d_{i j}-\eta h_{k, i} h_{k, j}-\zeta h_{i} h_{j}$

where $p$ is the hydrostatic pressure, $\mu$ is viscosity, $d_{i j}$ is rate of deformation tensor, $\eta$ is a coupling coefficient, $\zeta h_{i} h_{j}$ is the active stress term. The active stress represents the dipolar force generated by the individual particles, as a function of the director field. The sign of $\zeta$ determines whether the dipolar field generated by the particle is extensile $(\zeta>0)$ or contractile $(\zeta<0)$ [Edwards and Yeomans (2009)].

The cell nucleus was modeled as a hyperelastic material which was represented by a modified Mooney-Rivlin hyperelastic material model [Fried and Johnson (1988)]. The strain energy density function $W$ for this model is described [Zeng and Li (2011); Zeng and $\mathrm{Li}$ (2013); Lin and Zeng (2017)] as below:

$W=C_{1}\left(I_{1}-3 I_{3}^{1 / 3}\right)+C_{2}\left(I_{2}-3 I_{3}^{2 / 3}\right)+\frac{1}{2} \lambda\left(\ln I_{3}\right)^{2}$

where $C_{1}, C_{2}$ and $\lambda$ are material constants which determine the elastic properties of cell; $I_{1}, I_{2}, I_{3}$ are the invariants of the right Cauchy-Green tensor. Therefore, the second PiolaKirchhoff stress $\boldsymbol{S}$ can be expressed correspondingly as below [Lin and Zeng (2017)]:

$\boldsymbol{S}=2\left[\left(C_{1}+C_{2} I_{1}\right) \boldsymbol{I}-C_{2} \boldsymbol{C}-\left(C_{1} I_{3}^{1 / 3}+2 C_{2} I_{3}^{2 / 3}-\lambda \ln I_{3}\right) \boldsymbol{C}^{-1}\right]$

Here $\boldsymbol{C}=\boldsymbol{F}^{T} \boldsymbol{F}$ is the right Cauchy-Green deformation tensor. $\boldsymbol{P}$ is the first Piola-Kirchhoff stress tensor which can be derived from the second Pilola-Kirchhoff stress: $\boldsymbol{P}=\boldsymbol{S} \cdot \boldsymbol{F}^{T}$. In order to calculate the internal nodal force, $\boldsymbol{P}$ is substituted into the meshfree Galerkin formulation.

\section{Numerical simulation}

\subsection{Simulation model}

The cell diameter was assumed as $10 \mu \mathrm{m}$ based on experimental observations [Fedosov and Gompper (2014); Palmer, Briggs, McFadden et al. (2015)]. The volume ratio of nucleus to cell is around $6-8 \%$ based on literature data [Huber and Gerace (2007); Jorgensen, Edgington, Schneider et al. (2007)]. Then, we assumed the radius ratio of nucleus to cell as 0.283 . A total of 4,455 particles were used in discretization of cell in the meshfree computation. The meshfree dilation parameters are selected as $\rho_{x}=2.2 \Delta_{x}, \rho_{y}=$ $2.2 \Delta_{y}$. The initial cell density is set to be $1.0 \times 10^{3} \mathrm{~kg} / \mathrm{m}^{3}$. The material constants of the hyperelastic material model for cell nucleus were set as $C_{1}=2.126 \mathrm{KPa}, C_{2}=0.17 \mathrm{KPa}$, $\lambda=170 \mathrm{KPa}$ [Zeng and Li (2011); Ravetto, Wyss, Anderson et al. (2014); Lin and Zeng (2017)]. For cell plasma parameter, the viscosity is $\mu=0.001 \mathrm{~kg} /(\mathrm{ms})$ and the bulk modulus was set as $\mathrm{k}=2.2 \times 10^{6} \mathrm{KPa}$, the coupling coefficient is $\eta=5.0 \times 10^{-8}$. The active term was ignored in this work. The cell-cell adhesion bond density was set as $N_{c-c}=$ $1000\left(1 / \mu m^{2}\right)$ and the energy depth is selected as $\sigma=0.024(p N \cdot \mu m)$ for cell-cell 
adhesion, and the equilibrium position is $\varepsilon=10 \mathrm{~nm}$ [Roy and Qi (2010)]. The cellsubstrate ligand-receptor bond density was set as $N_{c-s}=8,000\left(1 / \mu m^{2}\right)$ and the energy depth is $\sigma=0.03(p N \cdot \mu m)$ for cell-substrate interaction. These parameter values are selected from published literature and they are in the suggested value range.

\subsection{Simulation results}

First, simulation was carried out when the protrusion force is generated at the leading edges of all three monocyte cells. The protrusion force was applied on the cell front half surface in contact with the substrate. The applied protrusion was proportional to the cell-substrate adhesion and we used a random number generator to adjust the value by multiplying it with a random number $\alpha=[0 \sim 1]$ at different locations. So the protrusion force is not constant at different locations. From this simulation, it can be observed that the cells are spreading over the substrate as shown in Fig. 2. We output the cell shape and effective stress of each cell at different time during the migration process, one may find that the contact area between cells and the substrate surface increased due to the cell-substrate adhesion (Figs. 2(a) \& 2(b)). After initial spreading, cells started to move over the substrate driven by protrusion force applied at the leading edges (Figs. 2(b) \& 2(c)). It can be seen that the contact areas between adjacent cells increased with time, which indicated that cells might exert lateral pushing on neighboring cells due to cell-cell interaction, resulting in cell displacement and movement [Haeger, Wolf, Zegers et al. (2015)].

(a)

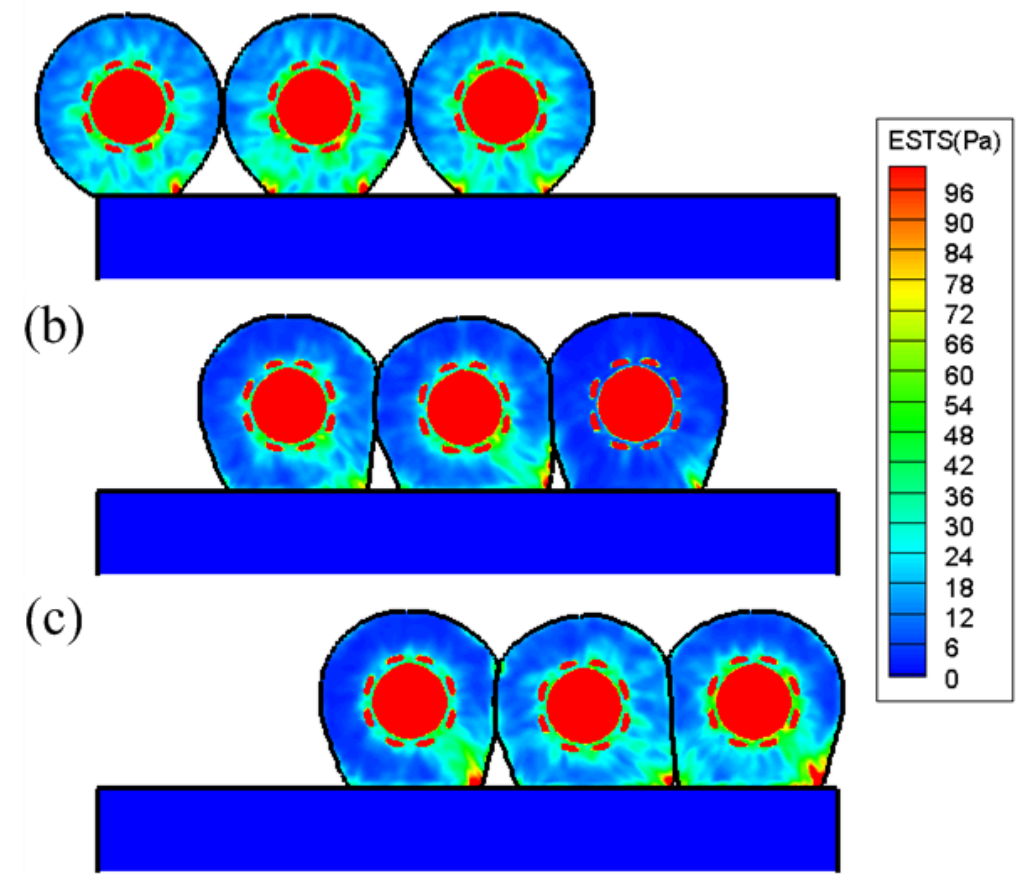

Figure 2: Snapshots of effective stress contour in cells (protrusion force applied to all cells) during collective cell migration: (a) $t=20 \mu \mathrm{s}$, (b) $t=204 \mu \mathrm{s}$, (c) $t=268 \mu \mathrm{s}$ 
We plotted the stress $\sigma_{11}$ at the lower center point of the middle cell during cell migration as shown in Fig. 3. One may observe that the stress in cell migratory direction oscillates. Such stress profile implies that pushing and pulling may be imposed by neighboring cells which is highly important during collective cell migratory process [Vedula, Leong, Lai et al. (2012)].

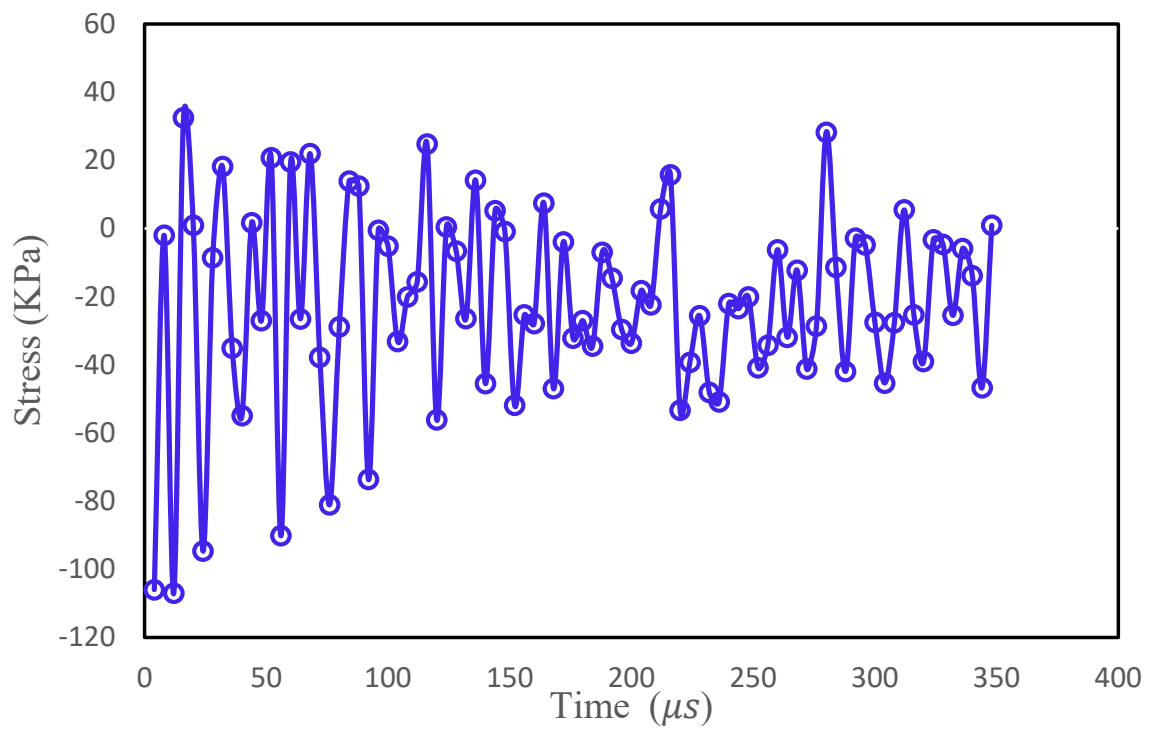

Figure 3: Stress status of the middle cell (Cell-2) during collective cell migration

In order to study how individual cells will affect collective migration behavior of the whole cell aggregate, three more cases were generated through applying protrusion force at the front edge of different number of monocyte cells. We output the average displacement of the threecell aggregate for all four cases as shown in Fig. 4. The results show that the migratory displacement is smallest when the protrusion force is only applied at the leading edge of Cell3 , while the displacement is largest when the protrusion forces are applied at the leading edges of all three cells. This might indicate that the number of leader cells in the cell aggregate plays a role in the collective migratory behavior. In addition, it can be observed that the displacement of the cell aggregate for the case in which Cell-1 and Cell-3 are self-propelled is smaller than that for the case in which Cell-2 and Cell-3 are self-propelled. The possible reason for the smaller displacement is that at the very beginning of the migration, Cell-2 and Cell-1 were separated so that it inhibits the communication between the two leader cells (Cell-1 \& Cell-3) as shown in Fig. 5(a).The results above shows that cell-cell interaction and communication between adjacent cells are very important and may regulate the collective cell migratory behavior [Khalil and Friedl (2010); Friedl and Mayor (2017)]. From the contour of effective stress shown in Fig. 5, one can find that the stress in Cell-2 is lower than the two self-propelled cells (Cell-1 \& Cell-3). This implies that the stress in the leader cell may be higher than the stress in the follower cell, which is in agreement with a recent study [Vishwakarma, Di Russo, Probst et al. (2018)]. 
We also preformed parametric studies in cell-cell adhesion strength. We found out that as the cell-cell adhesion strength increases, the collective cell migration speed increases as shown in Fig. 6. A parametric study in protrusion force indicated that the migration speed increases as the averaged cell protrusion increases.

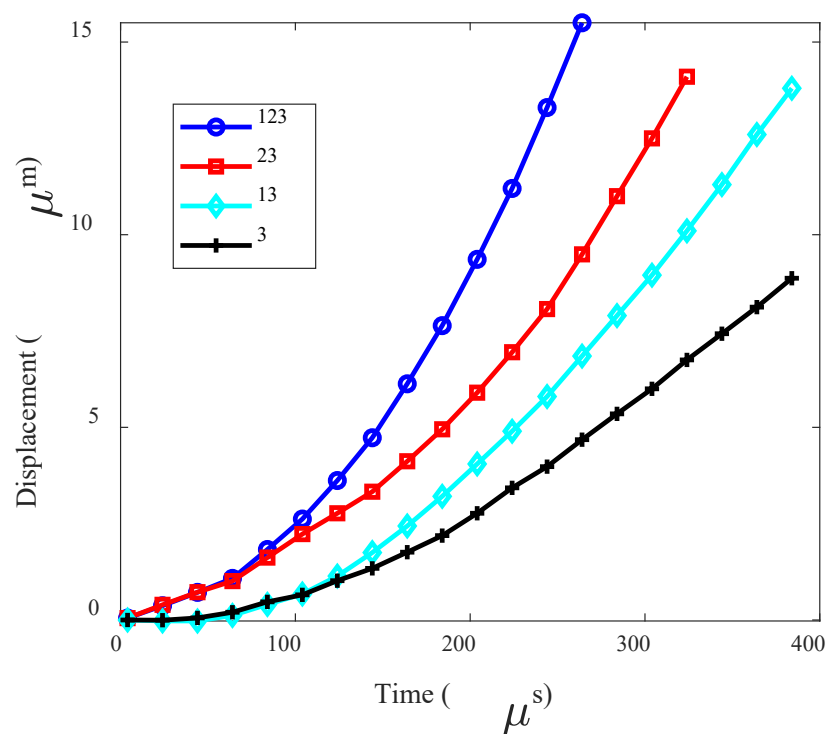

Figure 4: Average displacement of the cell aggregate during the collective cell migration (the number indicates which cell has protrusion force applied)

(a)

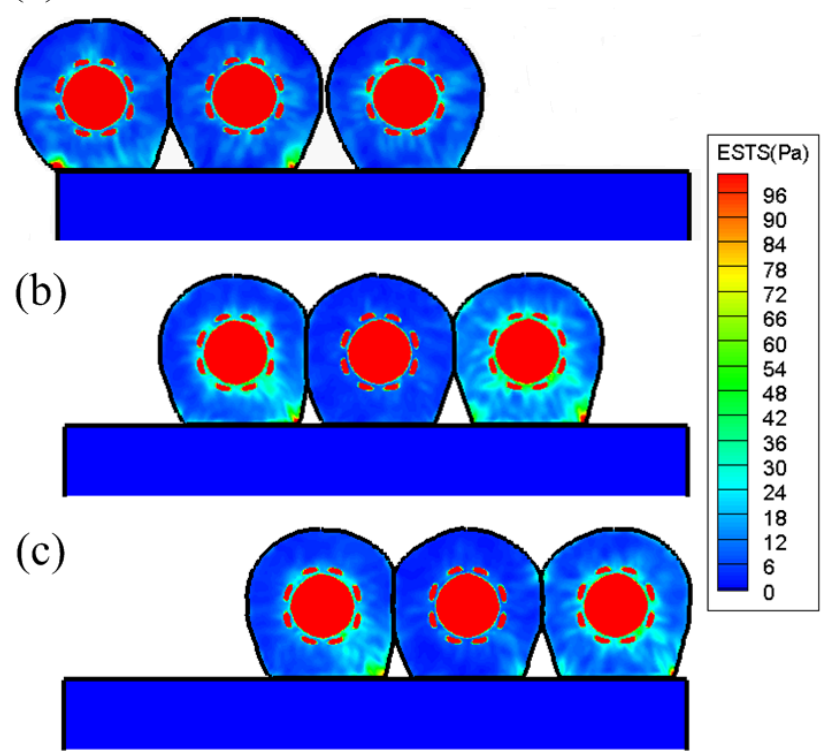

Figure 5: Snapshots of effective stress contour in cells during collective cell migration when Cell-1 \& Cell-3 generate protrusion force: (a) $t=24 \mu \mathrm{s}$, (b) $t=292 \mu \mathrm{s}$, (c) $t=388 \mu \mathrm{s}$ 


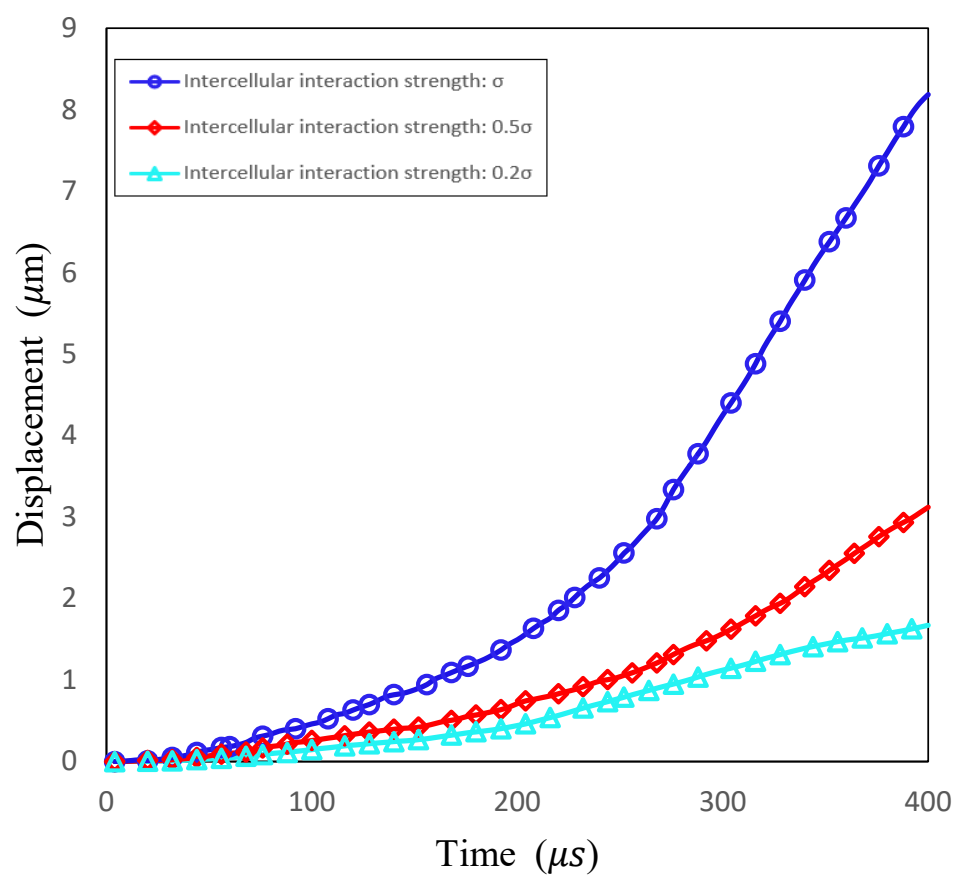

Figure 6: Average displacement of the cell aggregate with different magnitudes of cellcell adhesion strength

\section{Discussions and conclusions}

To develop continuum physics-based cell model system that is capable of explaining the coordinated cell motion is a complex and challenging task. In this work, the cell was modeled as nematic liquid crystal with hyperelastic cell nucleus. A coarse-grained potential force was proposed to model the cell-substrate and cell-cell interactions. The collective cell migration behavior on a substrate was studied by using RKPM meshfree method. Through this study, the pulling and pushing phenomenon can be observed during collective cell migration process. It was found that the number of self-propelled cells in the system significantly influenced the collective cell migratory behavior. The more self-propelled cells are in the system, the faster the collective cell migration is toward the coordinated direction. The model-based study may provide possible explanations and insights on coordinated cell motion at the cellular level.

It should be noted that the collective cell migration is a very complex biological process. The proposed model is only intended to model the mechanical interactions of cells at mesoscale level, which cannot explain the molecular mechanisms of cellular processes. The computational model presented in this work is a primitive $2 \mathrm{D}$ model without consideration of "chemotaxis" or "durotaxis" in migrating cells, so the protrusion force applied may not be realistic in real situation. In our future work, we plan to build 3D models. The current study may provide computational model and simulation tool for our future work and may open a door for the study of coordinated cell motion in more realistic settings. 
Acknowledgement: This work is supported by a grant from National Institutes of Health (Grant No. SC2GM112575) and a grant from the John L. Santikos Charitable Foundation of the San Antonio Area Foundation.

\section{References}

Bi, D.; Lopez, J.; Schwarz, J.; Manning, M. L. (2015): A density-independent rigidity transition in biological tissues. Nature Physics, vol. 11, no. 12, pp. 1074.

Brodland, G. W.; Viens, D.; Veldhuis, J. H. (2007): A new cell-based FE model for the mechanics of embryonic epithelia. Computer Methods in Biomechanics and Biomedical Engineering, vol. 10, no. 2, pp. 121-128.

Caille, N.; Thoumine, O.; Tardy, Y.; Meister, J. J. (2002): Contribution of the nucleus to the mechanical properties of endothelial cells. Journal of Biomechanics, vol. 35, no. 2, pp. 177-187.

Chen, H. H.; Brodland, G. W. (2000): Cell-level finite element studies of viscous cells in planar aggregates. Journal of Biomechanical Engineering, vol. 122, no. 4, pp. 394-401.

Dao, M.; Lim, C. T.; Suresh, S. (2003): Mechanics of the human red blood cell deformed by optical tweezers. Journal of the Mechanics and Physics of Solids, vol. 51, no. 11, pp. 2259-2280.

Edwards, S.; Yeomans, J. (2009): Spontaneous flow states in active nematics: a unified picture. Europhysics Letters, vol. 85, no. 1, pp. 18008.

Fan, H.; Li, S. (2015): Modeling universal dynamics of cell spreading on elastic substrates. Biomechanics and Modeling in Mechanobiology, vol. 14, no. 6, pp. 1265-1280.

Farhadifar, R.; Röper, J. C.; Aigouy, B.; Eaton, S.; Jülicher, F. (2007): The influence of cell mechanics, cell-cell interactions, and proliferation on epithelial packing. Current Biology, vol. 17, no. 24, pp. 2095-2104.

Farsad, M.; Vernerey, F. J. (2012): An XFEM-based numerical strategy to model mechanical interactions between biological cells and a deformable substrate. International Journal for Numerical Methods in Engineering, vol. 92, no. 3, pp. 238-267.

Fedosov, D. A.; Gompper, G. (2014): White blood cell margination in microcirculation. Soft Matter, vol. 10, no. 17, pp. 2961-2970.

Fletcher, A. G.; Osterfield, M.; Baker, R. E.; Shvartsman, S. Y. (2014): Vertex models of epithelial morphogenesis. Biophysical Journal, vol. 106, no. 11, pp. 2291-2304.

Frascoli, F.; Hughes, B. D.; Zaman, M. H.; Landman, K. A. (2013): A computational model for collective cellular motion in three dimensions: general framework and case study for cell pair dynamics. PLoS One, vol. 8, no. 3, e59249.

Fried, I.; Johnson, A. R. (1988): A note on elastic energy density functions for largely deformed compressible rubber solids. Computer Methods in Applied Mechanics and Engineering, vol. 69, no. 1, pp. 53-64.

Friedl, P.; Mayor, R. (2017): Tuning collective cell migration by cell-cell junction regulation. Cold Spring Harbor Perspectives in Biology, vol. 9, no. 4, a029199. 
Graner, F.; Glazier, J. A. (1992): Simulation of biological cell sorting using a twodimensional extended potts model. Physical Review Letters, vol. 69, no. 13, pp. 2013.

Haase, K.; Pelling, A. E. (2015): Investigating cell mechanics with atomic force microscopy. Journal of the Royal Society, Interface, vol. 12, no. 104, pp. 2014097020140970.

Haeger, A.; Wolf, K.; Zegers, M. M.; Friedl, P. (2015): Collective cell migration: guidance principles and hierarchies. Trends in Cell Biology, vol. 25, no. 9, pp. 556-566.

Helfrich, W. (1973): Elastic properties of lipid bilayers: theory and possible experiments. Zeitschrift für Naturforschung C, vol. 28, no. 11-12, pp. 693-703.

Honda, H.; Eguchi, G. (1980): How much does the cell boundary contract in a monolayered cell sheet? Journal of Theoretical Biology, vol. 84, no. 3, pp. 575-588.

Huber, M. D.; Gerace, L. (2007): The size-wise nucleus: nuclear volume control in eukaryotes. Journal of Cell Biology, vol. 179, no. 4, pp. 583-584.

Hwang, S. H.; Jung, S. H.; Lee, S.; Choi, S.; Yoo, S. A. et al. (2015): Leukocyte-specific protein 1 regulates T-cell migration in rheumatoid arthritis. Proceedings of the National Academy of Sciences, vol. 112, no. 47, pp. E6535-E6543.

Jorgensen, P.; Edgington, N. P.; Schneider, B. L.; Rupeš, I.; Tyers, M. et al. (2007): The size of the nucleus increases as yeast cells grow. Molecular Biology of the Cell, vol. 18, no. 9, pp. 3523-3532.

Karcher, H.; Lammerding, J.; Huang, H.; Lee, R. T.; Kamm, R. D. et al. (2003): A three-dimensional viscoelastic model for cell deformation with experimental verification. Biophysical Journal, vol. 85, no. 5, pp. 3336-3349.

Khalil, A. A.; Friedl, P. (2010): Determinants of leader cells in collective cell migration. Integrative Biology, vol. 2, no. 11-12, pp. 568-574.

Kruse, K.; Joanny, J. F.; Jülicher, F.; Prost, J.; Sekimoto, K. (2005): Generic theory of active polar gels: a paradigm for cytoskeletal dynamics. European Physical Journal E, vol. 16, no. 1, pp. 5-16.

Li, B.; Sun, S. X. (2014): Coherent motions in confluent cell monolayer sheets. Biophysical Journal, vol. 107, no. 7, pp. 1532-1541.

Li, S.; Liu, W. K. (2002): Meshfree and particle methods and their applications. Applied Mechanics Reviews, vol. 55, no. 1, pp. 1-34.

Li, S.; Liu, W. K. (2007): Meshfree Particle Methods. Springer Berlin Heidelberg.

Lin, F. H.; Liu, C. (2000): Existence of solutions for the Ericksen-Leslie system. Archive for Rational Mechanics and Analysis, vol. 154, no. 2, pp. 135-156.

Lin, L.; Zeng, X. (2017): Computational study of cell adhesion and rolling in flow channel by meshfree method. Computer Methods in Biomechanics and Biomedical Engineering, vol. 20 , no. 8 , pp. 832-841.

Lin, L.; Zeng, X. (2018): Numerical investigation of the role of intercellular interactions on collective epithelial cell migration. Biomechanics and Modeling in Mechanobiology, vol. 17, no. 2, pp. 439-448. 
Liu, P.; Zhang, Y.; Cheng, Q.; Lu, C. (2007): Simulations of the spreading of a vesicle on a substrate surface mediated by receptor-ligand binding. Journal of the Mechanics and Physics of Solids, vol. 55, no. 6, pp. 1166-1181.

Liu, W. K.; Li, S.; Belytschko, T. (1997): Moving least-square reproducing kernel methods (I) methodology and convergence. Computer Methods in Aapplied Mechanics and Engineering, vol. 143, no. 1-2, pp. 113-154.

McGarry, J. P.; Murphy, B. P.; McHugh, P. E. (2005): Computational mechanics modelling of cell-substrate contact during cyclic substrate deformation. Journal of the Mechanics and Physics of Solids, vol. 53, no. 12, pp. 2597-2637.

Mihai, L. A.; Wyatt, H.; Goriely, A. (2017): Microstructure-based hyperelastic models for closed-cell solids. Proceedings of Mathematical, Physical, and Engineering Sciences, vol. 473, no. 2200, pp. 20170036-20170036.

Mongera, A.; Rowghanian, P.; Gustafson, H. J.; Shelton, E.; Kealhofer, D. A. et al. (2018): A fluid-to-solid jamming transition underlies vertebrate body axis elongation. Nature, vol. 561, no. 7723, pp. 401-405.

Muller, W. A. (2013): Getting leukocytes to the site of inflammation. Veterinary Pathology, vol. 50, no. 1, pp. 7-22.

Munder, M. C.; Midtvedt, D.; Franzmann, T.; Nüske, E.; Otto, O. et al. (2016): A pHdriven transition of the cytoplasm from a fluid- to a solid-like state promotes entry into dormancy. eLife, vol. 5, e09347.

Orellana, R.; García-Solares, J.; Donnez, J.; Van Kerk, O.; Dolmans, M. M. et al. (2017): Important role of collective cell migration and nerve fiber density in the development of deep nodular endometriosis. Fertility and Sterility, vol. 107, no. 4, pp. 987995, e985.

Palmer, L.; Briggs, C.; McFadden, S.; Zini, G.; Burthem, J. et al. (2015): ICSH recommendations for the standardization of nomenclature and grading of peripheral blood cell morphological features. International Journal of Laboratory Hematology, vol. 37, no. 3, pp. 287-303.

Peetermans, J. A.; Matthews, E. K.; Nishio, I.; Tanaka, T. (1987): Particle motion in single acinar cells observed by microscope laser light scattering spectroscopy. European Biophysics Journal, vol. 15, no. 2, pp. 65-69.

Prost, J.; Jülicher, F.; Joanny, J. F. (2015): Active gel physics. Nature Physics, vol. 11, pp. 111.

Ragazzon, M. R. P.; Gravdahl, J. T.; Vagia, M. (2018): Viscoelastic properties of cells: modeling and identification by atomic force microscopy. Mechatronics, vol. 50, pp. 271-281.

Ravetto, A.; Wyss, H. M.; Anderson, P. D.; den Toonder, J. M.; Bouten, C. V. (2014): Monocytic cells become less compressible but more deformable upon activation. PLoS One, vol. 9, no. 3, e92814.

Rey, A. D. (2010): Liquid crystal models of biological materials and processes. Soft Matter, vol. 6, no. 15, pp. 3402-3429.

Roy, S.; Qi, H. J. (2010): A computational biomimetic study of cell crawling. Biomechanics and Modeling in Mechanobiology, vol. 9, no. 5, pp. 573-581. 
Ruiz-Herrero, T.; Estrada, J.; Guantes, R.; Miguez, D. G. (2013): A tunable coarsegrained model for ligand-receptor interaction. PLoS Computational Biology, vol. 9, no. 11, e1003274.

Sauer, R. A.; Li, S. (2007): An atomic interaction-based continuum model for adhesive contact mechanics. Finite Elements in Analysis and Design, vol. 43, no. 5, pp. 384-396.

Seifert, U. (1991): Adhesion of vesicles in two dimensions. Physical Review A, vol. 43, no. 12 , pp. 6803 .

Simon, S. I.; Green, C. E. (2005): Molecular mechanics and dynamics of leukocyte recruitment during inflammation. Annual Review of Biomedical Engineering, vol. 7, no. pp. 151-185.

Simpson, K. J.; Selfors, L. M.; Bui, J.; Reynolds, A.; Leake, D. et al. (2008): Identification of genes that regulate epithelial cell migration using an siRNA screening approach. Nature Cell Biology, vol. 10, no. 9, pp. 1027.

Sleep, J.; Wilson, D.; Simmons, R.; Gratzer, W. (1999): Elasticity of the red cell membrane and its relation to hemolytic disorders: an optical tweezers study. Biophysical Journal, vol. 77, no. 6, pp. 3085-3095.

Tambe, D. T.; Hardin, C. C.; Angelini, T. E.; Rajendran, K.; Park, C. Y. et al. (2011): Collective cell guidance by cooperative intercellular forces. Nature Materials, vol. 10, no. 6, pp. 469.

Thoumine, O.; Ott, A. (1997): Time scale dependent viscoelastic and contractile regimes in fibroblasts probed by microplate manipulation. Journal of Cell Science, vol. 110, no. 17, pp. 2109-2116.

Thoumine, O.; Ott, A.; Cardoso, O.; Meister, J. J. (1999): Microplates: a new tool for manipulation and mechanical perturbation of individual cells. Journal of Biochemical and Biophysical Methods, vol. 39, no. 1, pp. 47-62.

Vedula, S. R. K.; Leong, M. C.; Lai, T. L.; Hersen, P.; Kabla, A. J. et al. (2012): Emerging modes of collective cell migration induced by geometrical constraints. Proceedings of the National Academy of Sciences, vol. 109, no. 32, pp. 12974-12979.

Vernerey, F. J.; Farsad, M. (2011): A constrained mixture approach to mechano-sensing and force generation in contractile cells. Journal of the Mechanical Behavior of Biomedical Materials, vol. 4, no. 8, pp. 1683-1699.

Vishwakarma, M.; Di Russo, J.; Probst, D.; Schwarz, U. S.; Das, T. et al. (2018): Mechanical interactions among followers determine the emergence of leaders in migrating epithelial cell collectives. Nature Communications, vol. 9, no. 1, pp. 3469.

Vitorino, P.; Meyer, T. (2008): Modular control of endothelial sheet migration. Genes \& Development, vol. 22, no. 23, pp. 3268-3281.

Wu, Y.; Siadaty, M.; Berens, M.; Hampton, G.; Theodorescu, D. (2008): Overlapping gene expression profiles of cell migration and tumor invasion in human bladder cancer identify metallothionein $1 \mathrm{E}$ and nicotinamide N-methyltransferase as novel regulators of cell migration. Oncogene, vol. 27, no. 52, pp. 6679. 
Zeng, X.; Li, S. (2011): Modelling and simulation of substrate elasticity sensing in stem cells. Computer Methods in Biomechanics and Biomedical Engineering, vol. 14, no. 5, pp. 447-458.

Zeng, X.; Li, S. (2011): Multiscale modeling and simulation of soft adhesion and contact of stem cells. Journal of the Mechanical Behavior of Biomedical Materials, vol. 4, no. 2, pp. 180-189.

Zeng, X.; Li, S. (2012): A three dimensional soft matter cell model for mechanotransduction. Soft Matter, vol. 8, no. 21, pp. 5765-5776.

Zeng, X.; Li, S. (2013): Biomechanical cell model by liquid-crystal elastomers. Journal of Engineering Mechanics, vol. 140, no. 4, 04013003.

Zhu, C. (2000): Kinetics and mechanics of cell adhesion. Journal of Biomechanics, vol. 33, no. 1, pp. 23-33.

Zhu, C.; Bao, G.; Wang, N. (2000): Cell mechanics: mechanical response, cell adhesion, and molecular deformation. Annual Review of Biomedical Engineering, vol. 2, no. 1, pp. 189-226. 